\title{
An Active Fault Tolerant Control Strategy based on Bond Graph Adaptive Observers
}

\author{
Hajer Najari \\ MACS Laboratory \\ National Engineering School of Gabes \\ Gabes university, 6029 Tunisia
}

\author{
Rafika El Harabi \\ MACS Laboratory \\ National Engineering School of Gabes \\ Gabes university, 6029 Tunisia
}

\author{
Mohamed Naceur Abdelkrim \\ MACS Laboratory \\ National Engineering School of Gabes \\ Gabes university, 6029 Tunisia
}

\begin{abstract}
System reliability has become one of the major concerns on industrial systems survey in order to guarantee a system lifecycle far from malfunctions, disturbances and dangers. In this context, Fault Tolerant Control systems are involved in automation engineering issues as a remedy to system reliability troubles. Thereby, we deal in this paper with a graphical Active Fault Tolerant Control $(A F T C)$ law that compensates actuator fault effects, guarantees desirable closed-loop performances and system resilience. This tolerant control law is based on a linear Bond Graph $(B G)$ adaptive observer to detect and estimate failures. This control framework and the adaptive observer are designed by a graphical concept using Bond Graph tool as a useful methodology for multidisciplinary systems and which is based on structural, causal and behavioural properties. To emphasis proposed controller efficiency, an hydraulic system with two tanks is modelled and controlled with and without fault scenarios.
\end{abstract}

\section{General Terms}

Graphical Modelisation, Fault Tolerant Control.

\section{Keywords}

Bond Graph, Adaptive observer, Fault detection and estimation, Active Fault Tolerant Control, Feedforward control.

\section{INTRODUCTION}

Increasing system productivity is often the most desirable aim in industrial systems conception. However, human and system safety is also considered as one of the important pretention and it becomes a great challenge for automatical engineers. Thus, we have to confront any system malfunction which can be caused by system component's defaults or disturbances which can have bad consequences as system or products damage or human life threat. As a key solution for these great and several issues which threat system safety and may have critical and dangerous consequences especially in aeronautics, medicines and chemical or nuclear industry, we find Fault Tolerant Control system synthesis. This latter guarantees the total or the partial compensation of failure effects with maintain eligible system performances and essentially stability.

In literature, these FTC approaches can be further classified into two categories: passive approaches (as robust control) and active approaches (as adaptive control). These first control laws (PFTC) are designed for a limited number of faults and they did not need to have any estimation of failure magnitudes [11] [6]. However, the latter type $(A F T C)$ is designed on line and requires a fault estimation block so as to reconfigure control actions accordingly to failure magnitudes and natures and to system performances losses [19] [3]. Based on mathematical tools, we can find different fault tolerant laws as the Eigenstructure Assignment $(E A)$, the Pseudo Inverse Model (PIM), the predictive control, the Linear Quadratic $(L Q)$ approach or using the Linear Matrix Inequality ( $L M I)$,etc. [17]. Using these analytical tools, we can achieve failures accommodation task by modifying our classical control law or reconfiguration tasks when we have to modify both the control law and the system to be controlled in order to remedy system default and to attain acceptable performances. In this present work, we are concerned to deal with AFTC systems in order to compensate failure effects.

In addition to these analytical laws, we also find graphical ones which become more and more used in several engineering fields to diagnose [4] and to control [16] systems positing here some beneficial points for this use. Graphical approaches are often chosen for their physical signification and structural representation, their easy reading and interpretation using bonds and interconnections and exclusively because they emphasis cause-effect relations and power exchange through bonds. In other words, they show the dual exchanges of two power variables: effort and flow between system components. As graphical tools, we can find the bond graph used on our case, the hyper graph, informational graphs, Causal Temporal graphs and the signed directed graphs,etc.

The aim of this paper is to treat active fault tolerant control problem using graphical approaches based on bond graph representation. This investigated strategy is founded on a feedforward control to reach the steady-state tracking and inversal bond graph model to maintain system performances nearly to those in faulty free case and to accommodate faults. The proposed approach is established using a graphical adaptive observer based on bond graph and which is used to detect and estimate failures. In the present work, only actuator faults are considered.

The outline of this work is structured such as: Section II presents some previous results concerning fault tolerance as related works. In section III, the proposed AFTC strategy is given. In this part, a flashback to our graphical adaptive observer used to detect and estimate fault is addressed. Afterward, the efficiency of the developed algorithm is shown by means of an hydraulic system in Section IV 
with a discussion part that analyzes obtained results and compares them to those when we use an additive control law with a graphical PI controller. At last, Section V draws some remarks as a conclusion.

\section{RELATED WORKS}

In literature, we can find several approaches concerning graphical fault tolerant control that give satisfying results. As graphical passive control law, we can highlight the work done by Nacusse et al. [8] using $B G$. The proposed approach was based on an energy and power shaping strategy. So they proposed to consider control system parameters in terms of desired closed-loop energy and power dissipation functions using a graphical representation called Target Bond Graph $(T B G)$. This $T B G$ model can be considered as a reference model. Then, we talk here about modified Diagnostic Bond Graph $(m D B G)$ to generate a residual signal which is the error between the desired and the actual control system performances. If this residual signal becomes equal to zero, we can say that the diagnosed system and the $T B G$ have similar behaviours and then we can say that control tasks are achieved. As graphical active approaches, we can say that most investigated works deal with inversal Bond Graph in order to solve tolerant control problem using controller reconfiguration. That means to find a novel system input appropriate to our faulty system in order to achieve desirable or nominal performances. Noting that to achieve system inversion graphically we need to use bicausal bond graph properties. In this case, we notice the use of system inversion in combination with the Linear Fractional Transformation method $(B G-L F T)$ in [7] used before to estimate failures magnitude. This inversal BG model was used also by Borutzky [2] in order to compensate failures involving Analytical Redundancy Relations ( $A R R$ ) approach to calculate the desired input. This strategy aims to reconfigure control law to depending on fault scenarios and with the given hypothesis adopted to consider a case where a single fault can be isolated and estimated. To avoid isolation and estimation tasks used often before fault compensation, Allous [1] have proposed to use a classical Luenberger observer based on ARRs calculus that gives such residues. The latter becomes then an input to the inversal $B G$ model.

\section{PROPOSED FTC STRATEGIES}

For diagnosis tasks, we can find multi graphical tools illustrated in literature but we are interested here by graphical observer design to detect, isolate and estimate actuator failures [13] [15] [5]. Based on an analytical approach proposed by K. Zhang et al. [17], we have synthesized in previous work a graphical observer that deals with fault detection and estimation for linear models [9]. The elaborated approach was based on an adaptive estimation using a Luenberger observer as it will be designed in the sequel. Considering the linear model (1):

$$
\left\{\begin{array}{l}
\dot{x}(t)=A x(t)+B u(t)+E f(t) \\
y(t)=C x(t)
\end{array}\right.
$$

where $x \in \Re^{n}$ is the state vector, $u \in \mathfrak{R}^{q}$ represents the set of input variables, $y \in \mathfrak{R}^{p}$ is the set of output variables and $f \in \mathfrak{R}^{r}$ is the set of fault variables (actuator faults in our case). A, B and E are known parameter matrices and supposed to be of full rank.

Some assumptions are required for the state space model $\sum(C, A, E)$.

Assumption 1. Its supposed that system defined in (1) is observable.

Assumption 2. Invariant zeros of system lie in the open left half plane.

To detect this fault, an adaptive observer is constructed in [17] as follows:

$$
\left\{\begin{array}{l}
\dot{\hat{x}}(t)=A \hat{x}(t)+B u(t)+K(y(t)-\hat{y}(t))+E \hat{f}(t) \\
\hat{y}(t)=C \hat{x}(t)
\end{array}\right.
$$

where $\hat{x} \in \Re^{n}$ is the state vector of observer, $\hat{y} \in \Re^{n}$ is the output vector of the observer and $\hat{f} \in \Re^{n}$ is an estimate of the actuator fault $f(t) . K \in \mathfrak{R}^{n \times p}$ is a selected gain matrix such that $(A-L C)$ is a stable matrix.

The errors vectors are defined, respectively according to the output $y(t)$ and the fault $f(t)$ :

$$
\begin{aligned}
& e_{y}(t)=\hat{y}(t)-y(t) \\
& e_{f}(t)=\hat{f}(t)-f(t)
\end{aligned}
$$

To achieve the fast fault estimation using the adaptive observer, we have to respect the following theorem :

Theorem 1: If there exist symmetric positive definite matrices $P, Q \in \Re^{n \times n}$, an observer gain $K \in \mathfrak{R}^{n \times p}$ and a matrix $F \in \Re^{r \times p}$ which check up the following conditions:

$$
\begin{gathered}
P(A-L C)+(A-L C)^{T} P=-Q \\
E^{T} P=F C
\end{gathered}
$$

then we can admit that the fault evolution is:

$$
\dot{\hat{f}}(t)=-\psi F e_{y}(t)
$$

Note $\psi \in \mathfrak{R}^{r \times r}$ is the learning rate matrix. Reaching the last theorem and from the last expression (8), we get the adaptive estimation of the actuator fault that can be written as :

$$
\hat{f}(t)=-\psi F \int_{t}^{t_{f}} e_{y}(\tau) d \tau
$$

Further, in view of the previous expression, the estimation of the actuator fault magnitudes can be achieved with a little delay in the estimation task. Nevertheless this latter which appears in the estimation step may affect fault effects compensation. This is why, Zhang et al. in [17] has adopted another expression that guarantees a fast adaptive fault estimation reaching theorem 2 .

Theorem 2. Given scalars $\sigma, \mu \succ 0$ if there exist symmetric positive definite matrices $P \in \mathfrak{R}^{n \times n}, Q \in \mathfrak{R}^{r \times r}$ and matrices $K \in \mathfrak{R}^{n \times p}$ , $F \in \mathfrak{R}^{r \times p}$ satisfying the two following conditions:

$\left[\begin{array}{ll}P A+A^{T} P-P K C-C^{T}(P K)^{T} & -\frac{1}{\sigma}\left(A^{T} P E-C^{T}(P K)^{T} E\right) \\ -\frac{1}{\sigma}\left(A^{T} P E-C^{T}(P K)^{T} E\right) & -2 \frac{1}{\sigma} E^{T} P E+\frac{1}{\sigma \mu} G\end{array}\right] \prec 0$

$$
E^{T} P=F C
$$

then we obtain the fault variation given by :

$$
\dot{\hat{f}}(t)=-\psi F\left(\dot{e}_{y}(t)+\sigma e_{y}(t)\right)
$$

From this last theorem, we can write the fast adaptive estimation $\hat{f}(t)$ for our actuator fault $f(t)$ as :

$$
\hat{f}(t)=-\psi F\left(e_{y}(t)+\sigma \int_{t}^{t_{f}} e_{y}(\tau) d \tau\right)
$$


where $\psi \in \mathfrak{R}^{r \times r}$ is the learning rate matrix. $F$ is given from the Linear Matrix Inequality $(L M I)$ resolution whereas $e_{y}$ is the residual vector.

Note that the first obtained result (see equation (8)) is only appropriate for identifying constant faults. Whereas equation (12) is improved and applied for time-varying faults [17]. In addition, we can affirm that added gain with the proportional part given by theorem 2 , in fault estimation, to obtain with the residue integration given by theorem 1 ameliorate considerably estimation fastness.

The adaptive graphical observer introduced in [9] is deducted automatically from the $B G$ based representation where the causality is in preferred integral form. Thereafter, the model observability is graphically verified by means of the next definition.

Definition 1. The bond graph model is observable if and only if the necessary conditions cited below are satisfied [12]: there exists a causal path between each dynamic component and one of the output detectors; each dynamic component is able to have causality in a preferred derivative form corresponding to the $B G$ description without facing any conflict.

Afterwards, the checking of the structural observability, the associated $B G$ observer model is developed founded on the bond graph representation. Thereby, one requires to inject the outputs linearly on each dynamical element of the observer-based $B G$ model by utilizing the modulated sources as shown in Fig.1. The observer gains are thus determined via both pole placement and $L M I$ resolution methods so as to perform the detection and adaptive estimation of occurred faults in the present case [12].

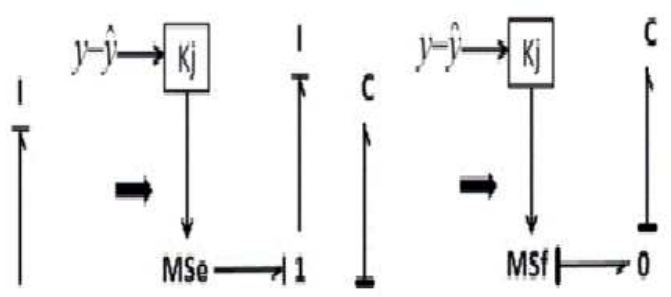

Fig. 1. Output linear injection.

In our previous work [10], we have synthesized a graphical AFTC using a Proportional Integral (PI) controller and an additive control law which is a Pseudo Inverse Model (PIM) law that compensates failure effects [14] calling out the graphical observer above mentioned to estimate fault magnitudes. The tolerant law used can be expressed as :

$$
u_{F T C}(t)=u_{\text {nom }}(t)+u_{\text {add }}(t)
$$

where $u_{\text {FTC }}(t)$ is the new control law, $u_{\text {nom }}(t)$ is the synthesized control law in nominal case to fulfill system performances given in this case by the graphical PI controller and $u_{\text {add }}(t)$ is the additive law to be elaborated in faulty case to reestablish nominal performances. As well, $\mathrm{u}_{\text {add }}$ is computed such that additive fault effects can be compensated. Hence, the following condition must be checked up :

$$
B u_{a d d}(t)+E f(t)=0
$$

Knowing that matrix $B$ must be of full rank. Given the fault estimation afforded by the graphical observer described herein, this additive control law can be written as :

$$
u_{\text {add }}(t)=-B^{+} E \hat{f}(t)
$$

where $B^{+}$is the pseudo-inverse of the control matrix $B$ calculated as following :

$$
B^{+}=\left[B^{T} B\right]^{-1} B^{T}
$$

$B^{T}$ is the matrix transpose.

From Fig. 2 and Fig.3, we can see that the PI controller is often used in control closed-loop to maintain the tracking error near or equal to zero. To implement this regulator, a flow source as the system input is the flow (or an effort source when the system input is an effort), a 1-junction (respectively 0 -junction) which represents the gap between the reference input and the output signal, a resistive element as a proportional part and an inertial one as integral part. The different parameters are calculated generally according to different characteristics of the $B G$ theory (the structure and causality aspects).

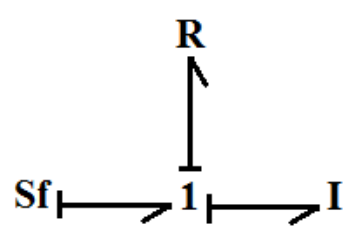

Fig. 2. Graphical PI controller with flow source input.

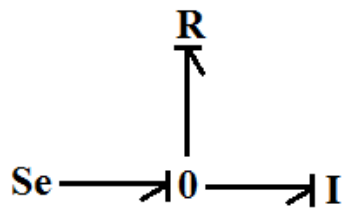

Fig. 3. Graphical PI controller with effort source input.

Remembering here that before any control law synthesize, we must check system structural controllability as it's mentioned in Property 1.

Property 1.: To say that a $B G$ model in a preferred integral causality is controllable, we must pay attention to the two following conditions:

- if there exists at least a causal path between each dynamical component $I$ or $C$ and one of the controlled sources $M S e$ or $M S f$.

- if each dynamical component $I$ or $C$ admit a derivative causality in preferred derivative bond graph model.

Here in our present work, we have synthesized a graphical AFTC law using the inversal bond graph notion instead of the pseudo inverse matrix. The inversal bond graph is a useful tool to control systems graphically after checking each time either the system is invertible or not as it is mentioned in proposition 1 in the sequel. The inversal system concept is founded on the use of an appropriate input to generate the desired output. This suitable input here is calculated through bicausality assignment on bond graph model. 
Proposition 1 : A linear system modeled by bond graph is invertible if there exist at least one causal path between the input variable and the output one.

To put forward this control law, we have called up some other previous work dealing with fault tolerance done by Zhang et al. [19] and Dongsheng et al. [3]. To achieve fault accommodation task, Zhang et al. [18] have synthesized a tolerant control law which is based on a feedback control part in addition to a feedforward control strategy. The feedback block is used to restore closed-loop system performances using the classical Eigenstructure Assignment $(E A)$. While the feedforward block is used to reach the steady-state tracking. The tolerant control law suggested can be then written as (17):

$$
u_{F T C}(t)=K_{\text {forward }} y_{\text {ref }}-K_{\text {feedback }} x
$$

where $y_{\text {ref }}$ is the reference input. To improve tolerance task, Zhang et al. [19] have ameliorated the last expression as (18):

$$
u_{F T C}(t)=K_{\text {forward }} y_{\text {ref }}+K_{\text {feedback }}\left(x-x^{*}\right)
$$

Taken the desired state value $x^{*}$ considered as a reference. The feedback control is also used by Dongsheng et al. [3] to tolerate faults as below:

$$
u_{F T C}(t)=-K_{\text {feedback }} x+y_{\text {ref }}-\hat{f}(t)
$$

Noting here that $\hat{f}(t)$ is the estimation of the considered fault. Taking account to these previous expressions, our proposed tolerant control law can be written as next :

$$
u_{F T C}(t)=K_{\text {forward }}\left(y_{\text {ref }}-y\right)+K_{\text {forward }} y_{\text {ref }}-K_{\text {forward }} \hat{f}(t)
$$

$\mathrm{K}_{\text {forward }}$ is a gain of the inverse Bond Graph model. $y_{\text {ref }}$ is our input reference, $y$ is our system output and $\hat{f}$ is an estimation for our additive actuator fault given by the observer. We have to point out here that this estimation should be accurate and fast in order to achieve acceptable performances through the control task.

\section{CASE STUDY : AN HYDRAULIC SYSTEM}

In order to exemplify these previous theoretical results, we consider an hydraulic system. As we can see from Fig.4, the chosen system is composed of two tanks $C_{1}$ and $C_{2}$ in series connected by valve $R_{1}$. The first tank $C_{1}$ is feeded by a controlled pump modeled as a source of a flow $M S f: F_{i}$. If the valve $R_{1}$ is opened, the fluid flows from the first tank to the second one.

The $B G$ model is depicted by Fig.5. From this model, we can check, easily, that our system is observable and controllable.

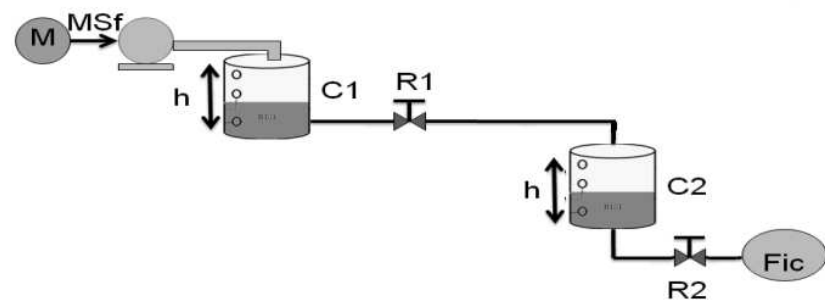

Fig. 4. Synoptic scheme of the real process.

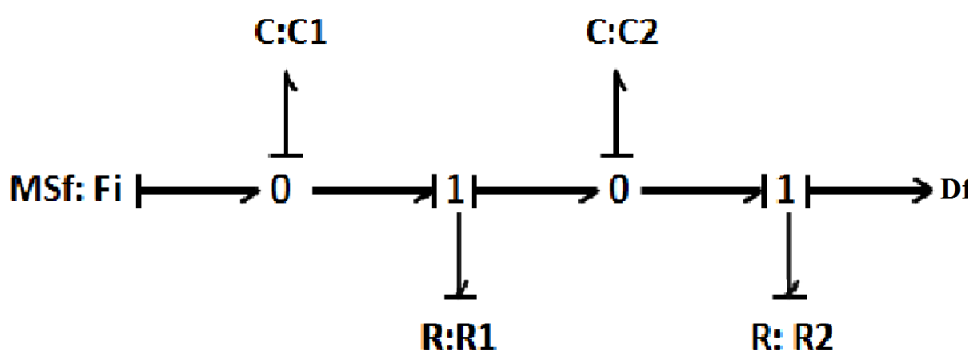

Fig. 5. Bond graph model in preferred integral causality.

Table 1. Numerical values of system parameters

\begin{tabular}{|c|c|c|c|}
\hline$C_{1}$ & $R_{1}$ & $C_{2}$ & $R_{2}$ \\
\hline $0.03 \mathrm{~m}^{2}$ & $1 \mathrm{~m}\left(\mathrm{~m}^{2} s^{-1}\right)^{-1}$ & $0.03 \mathrm{~m}^{2}$ & $1 \mathrm{~m}\left(\mathrm{~m}^{2} s^{-1}\right)^{-1}$ \\
\hline
\end{tabular}

Calculating the different structural laws and the gain for each element, the state space representation of this system can be written as (21) where $\left[x_{1}, x_{2}\right]^{T}$ is the state vector, $u=F_{i}$ is the input flow vector and $\left[y_{1}, y_{2}\right]^{T}$ is the output vector which corresponds to the flow delivered by each valve.

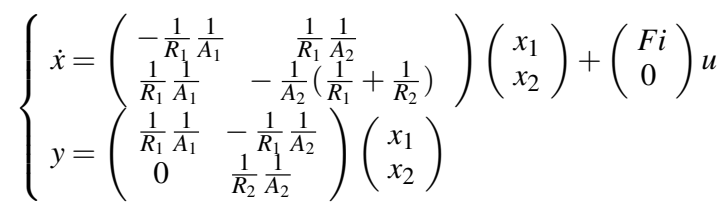

The hydraulic system is subject to an actuator fault $f(t)$ in our pump characteristics. This failures is here represented by an additive actuator fault. The new state equation of the faulty bond graph model is written as (22):

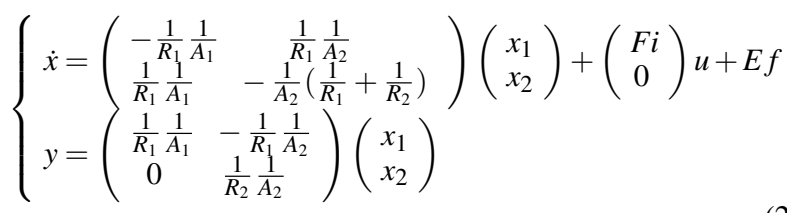

The numerical values of system parameters are shown in Table 1 . The control input (the inlet pump flow) is $u(t)=2.25 \times 10^{-4} \mathrm{~m}^{3} / \mathrm{s}$ and initial conditions are equal to 0 .

The considered actuator fault is an additive one represented by a pulse signal during $3 \mathrm{~s}$ (from $\mathrm{t}=3 \mathrm{~s}$ to $\mathrm{t}=6 \mathrm{~s}$ ) with an amplitude of $-0.5 \times 10^{-4} \mathrm{~m}^{3} / \mathrm{s}$.

To detect and estimate this failure, the adaptive observer is designed graphically as illustrated in Fig. 6 Where we find four different gain values. Gain 1 and Gain 2 are linked to the residue $e_{y}$ as any other classical Luenberger observer and they are calculated using poles placement technique. Whereas, Gain 3 and Gain 4 are obtained from the $L M I$ resolution.

Therefore, the observer proportional gains are calculated as Gain $1=K_{1}=0.01$ and Gain $2=K_{2}=2.99$.

The Gain 3 and Gain 4 are respectively equal to 0.2 and 0.025 . 


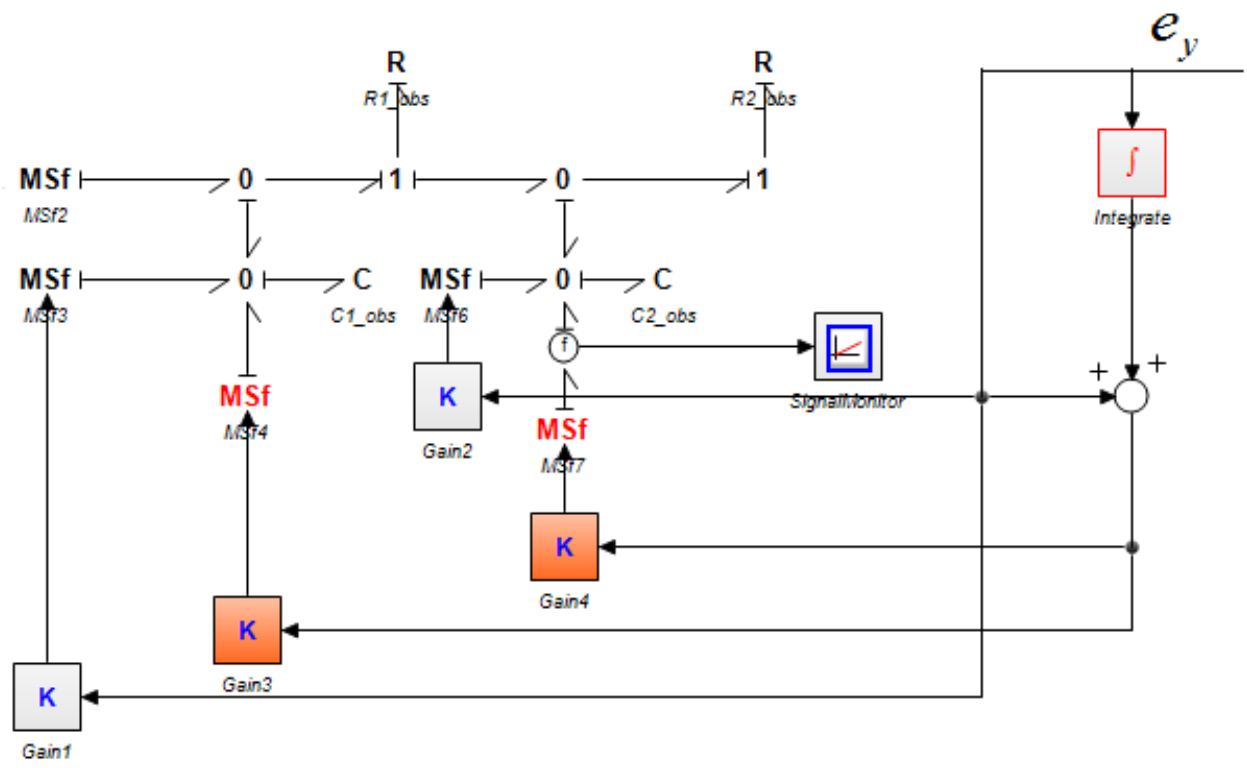

Fig. 6. Adaptive Luenberger observer bond graph model.

The fast fault estimation obtained is here compensated using graphical tolerant control laws ; starting with illustration of previous results given in [10]. As it is mentioned above, we study the liquid flow evolution so we deal with a flow parameter. Thus, we have used a graphical PI as illustrated in Fig. 3 integrated to the additive law. Simulation results are given by Fig. 12. Then, we deal with fault accommodation using inversal bond graph model to elaborate the proposed tolerant control strategy given by (20). Control loop blocks are illustrated by Fig.7 and simulation results as depicted next in Fig. 13.

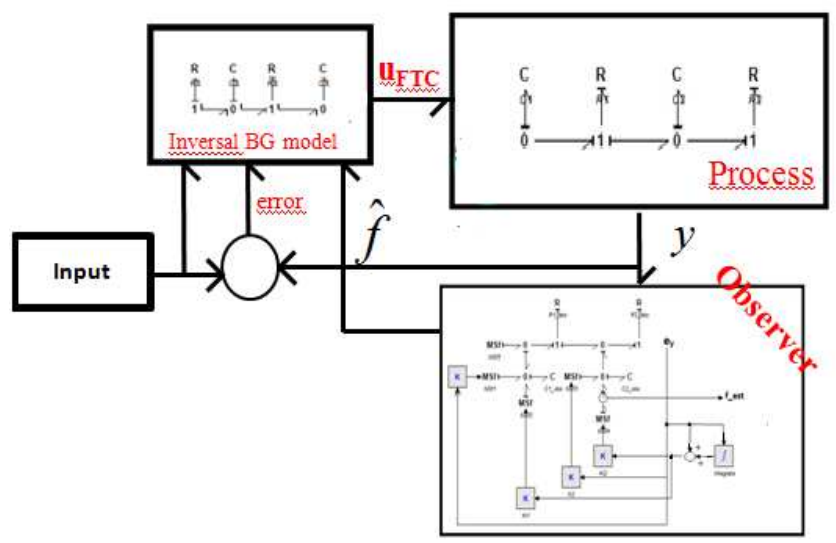

Fig. 7. Closed-loop system scheme.

Our system output evolution in nominal case is given by Fig. 8 . From Fig 10, it can be seen that the residual signal response is different from zero when the fault occurred. Therefore the actuator fault is detected. The real trajectory is very close to its estimate as

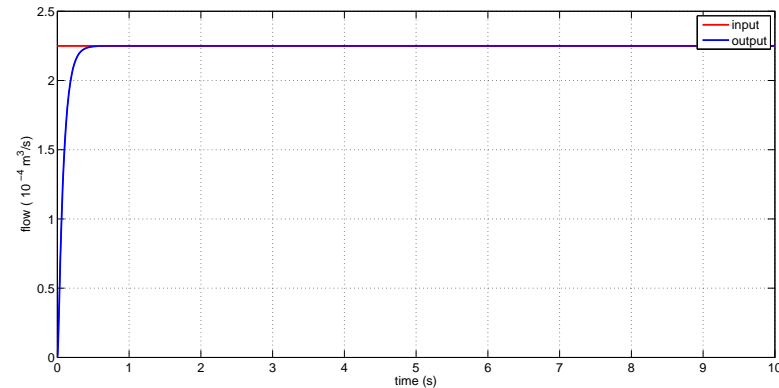

Fig. 8. Output signal in fault-free case.

seen in Fig 11 and this graphical observer allows a fast estimation of the actuator fault signal.

Our system output evolution in an actuator fault situation is shown by Fig.9.

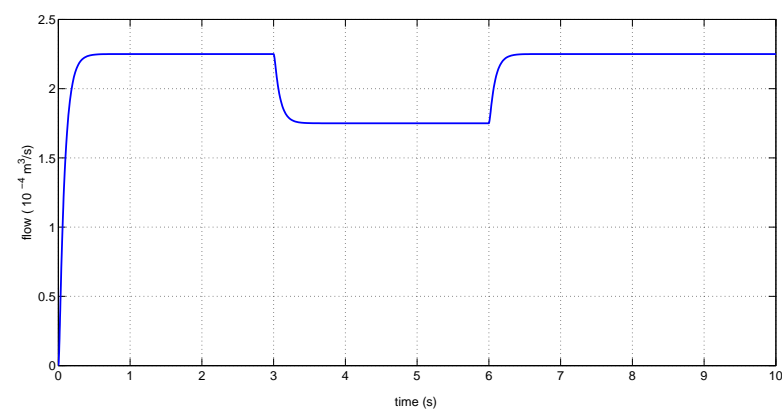

Fig. 9. Output signal in faulty case. 
Fault detection and estimation are illustrated by figures Fig.10 and Fig.11.

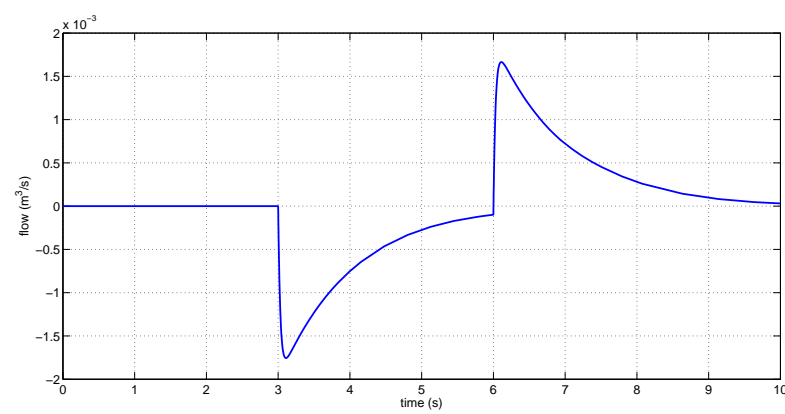

Fig. 10. Residual signal.

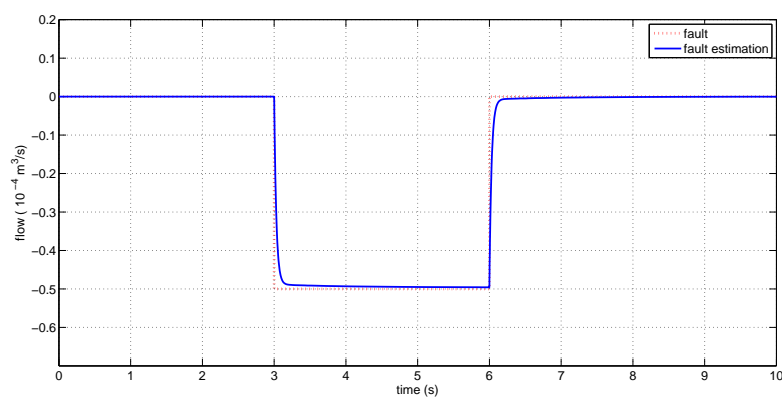

Fig. 11. Fault signal and its estimate.

Simulation results of our system behaviour without and with control loop are given by figures 9,12 , and 13 .

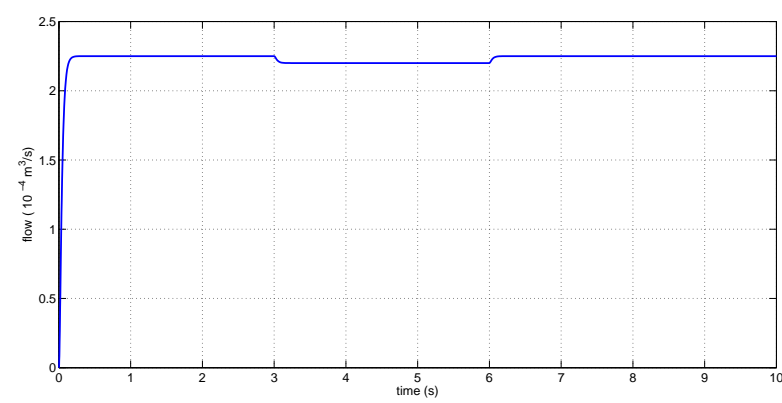

Fig. 12. Output signal in presence of graphical PI and additive control law.

From Fig.10, it can be observed that the fault indicator response is different from zero which reveal that our fault occurred and then detected. We can affirm that the used observer gives an accurate estimation as it can be seen from Fig. 11 where real trajectory is very close to its estimate. These results prove also a fast fault estimation which is intended in order to achieve efficient active tolerance task. Noting that this observer model can be used for constant or time-varying actuator fault. Active fault tolerant control has been here achieved, firstly, through additive control law and graphical PI

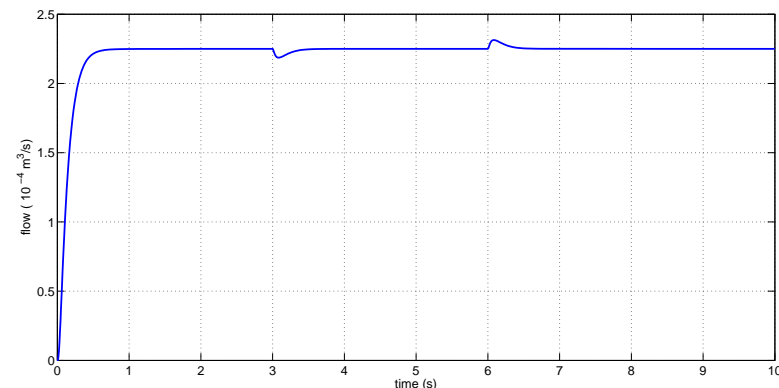

Fig. 13. Output signal in presence of the feedforward control and inversal bond graph.

control which ensure system performances restoration as it can be concluded from a simple comparison between Fig.12 and Fig.11 remembering here that this PI controller can be inserted directly in control loop from some software tool boxes or synthesized as mentioned in section III. Secondly, a feedforward control loop is elaborated based on inversal bond graph model which is used often in classic graphical control. This suggested law compensates failure effects as it is depicted by Fig. 13 and provide desirable performances due to feedforward strategy which solve steady-state tracking. Comparing results simulation of both control systems applied for the hydraulic system, we can remarkably see that the second law ensure better results to attenuate actuator fault. Moreover we can assume that both of them can guarantee accurate responses and system stability which is a critical point in control and especially when we talk about actuator malfunctions or saturation.

\section{CONCLUSION}

In this paper a graphical Active Fault Tolerant Control system has been investigated well-founded on bond graph concept to accommodate an actuator failure. This latter system malfunction was detected and fast estimated through a graphical adaptive observer. This synthesized law has been based on a feedforward control strategy that ensure the steady-state tracking and an inversal bond graph model using bicausality assignment to compensate failure effects. The relevance and efficiencies of this proposed control methodology are compared to those of a graphical PI controller integrated with an additive control law proposed in our previous work. From simulation results, we can see clearly that failure effects are compensated using both tolerant laws. Furthermore, acceptable closedloop system performances are reached in spite of some limitations such as necessary time to fault estimation and we have to point out that fault estimation must be fast and accurate to achieve better accommodation.

At last, we have to point out that in this paper we treat a system with single actuator fault to make fault detection and identification task easier. Sensor or component faults treatment or system with multiple faults case can be the purpose for forthcoming works.

\section{ACKNOWLEDGMENT}

This work was supported by the Ministry of the Higher Education and Scientific Research in Tunisia. 


\section{REFERENCES}

[1] Manel Allous and Nadia Zanzouri. Active fault tolerant control based on bond graph approach. Advances in Electrical Engineering, 2014, 2014.

[2] W Borutzky. Bond graph model-based fault accommodation in power electronic systems. In Control and Automation (MED), 2015 23th Mediterranean Conference on, pages 6875. IEEE, 2015.

[3] Dongsheng Du, Bin Jiang, and Peng Shi. Fault estimation and accommodation for switched systems with time-varying delay. International Journal of Control, Automation and Systems, 9(3):442-451, 2011.

[4] Rafika El Harabi, Belkacem Ould-Bouamama, and Mohamed Naceur Abdelkrim. Bond graph modeling for fault diagnosis: the continuous stirred tank reactor case study. Simulation, 90(4):405-424, 2014.

[5] I Gahlouz, E Tarasov, C Sueur, and B Ould Bouamama. Bond graph uio approach for fault components monitoring. In Control and Fault-Tolerant Systems (SysTol), 2013 Conference on, pages 73-78. IEEE, 2013.

[6] Jin Jiang and Xiang Yu. Fault-tolerant control systems: A comparative study between active and passive approaches. Annual Reviews in control, 36(1):60-72, 2012.

[7] Rui Loureiro, Rochdi Merzouki, and Belkacem Ould Bouamama. Bond graph model based on structural diagnosability and recoverability analysis: Application to intelligent autonomous vehicles. IEEE Transactions on Vehicular Technology, 61(3):986-997, 2012.

[8] M Nacusse and Sergio J Junco. Passive fault tolerant control: A bond graph approach. In Proceedings of the 5th International Conference on Integrated Modelling and Analysis in Applied Control and Automation (IMAACA 2011), DIPTEM Università di Genova, Rome, Italy, pages 75-82, 2011.

[9] Hajer Najari, Rafika El Harabi, and Mohamed Naceur Abdelkrim. Actuator faults estimation using adaptive luenberger bond graph observer. In Electrical Sciences and Technologies in Maghreb (CISTEM), 2014 International Conference on, pages 1-5. IEEE, 2014.

[10] Hajer Najari, Rafika El Harabi, and Mohamed Naceur Abdelkrim. A graphical active fault tolerant control approach. In Sciences and Techniques of Automatic Control and Computer Engineering (STA), 2015 16th International Conference on, pages 285-290. IEEE, 2015.

[11] Henrik Niemann and Jakob Stoustrup. Passive fault tolerant control of a double inverted penduluma case study. Control engineering practice, 13(8):1047-1059, 2005.

[12] César Pichardo-Almarza, Ahmed Rahmani, Geneviève Dauphin-Tanguy, and Marisol Delgado. Proportional-integral observer for systems modelled by bond graphs. Simulation Modelling Practice and Theory, 13(3):179-211, 2005.

[13] Ghada Saoudi, Rafika El Harabi, and Mohamed Naceur Abdelkrim. Graphical linear observers for fault detection: The dc motor case study. In Systems, Signals \& Devices (SSD), 2013 10th International Multi-Conference on, pages 1-8. IEEE, 2013.

[14] M Staroswiecki. Fault tolerant control: the pseudo-inverse method revisited. IFAC Proceedings Volumes, 38(1):418-423, 2005.
[15] E Tarasov, I Gahlouz, C Sueur, and B Ould Bouamama. Bond graph uio approach for fault components monitoring. In Control and Fault-Tolerant Systems (SysTol), 2013 Conference on, pages 257-262. IEEE, 2013.

[16] T-J Yeh. Controller synthesis for cascade systems using bond graphs. International Journal of Systems Science, 33(14):1161-1177, 2002.

[17] Ke Zhang, Bin Jiang, and Vincent Cocquempot. Adaptive observer-based fast fault estimation. International Journal of Control, Automation, and Systems, 6(3):320-326, 2008.

[18] YM Zhang and Jin Jiang. Active fault-tolerant control system against partial actuator failures. IEE proceedings-Control Theory and applications, 149(1):95-104, 2002.

[19] Youmin Zhang and Jin Jiang. Fault tolerant control system design with explicit consideration of performance degradation. IEEE Transactions on Aerospace and Electronic Systems, 39(3):838-848, 2003. 